Int. J. Contemp. Math. Sci., Vol. 1, 2006, no. 9, 419-438

\title{
Bayesian Approach to Estimation of Accuracy in Two-sided Testing
}

\author{
Hsiuying Wang \\ Institute of Statistical Science \\ Academia Sinica \\ Taipei 115, Taiwan
}

\begin{abstract}
For a two-sided hypothesis testing, it is known that there does not exist UMP tests. Uniformly most powerful unbiased (UMPU) tests and likelihood ratio test are the common approaches to two-sided testing. The p-values of these tests are usually used as evidence against null hypothesis. However, there are criticisms of p-values as a measure of evidence against null hypothesis for the two-sided testing problem in literature. Thus, in this paper, evidence measures derived from Bayesian approach are proposed to be the replacements of p-values and are investigated from both decision and testing perspectives. From decision theoretic framework, the proposed evidence measures can be demonstrated as admissible estimators, however, p-value of UMPU test are not admissible estimators; from testing aspect, the tests derived from p-values and the proposed evidence measures are shown to be UMPU tests. The Bayes estimator is better than p-value from theoretical aspect and it has the same merit as the p-value in testing point of view. Therefore, evidence measures derived from Bayesian approach are recommended in this paper.
\end{abstract}

Keywords: UMPU tests; Bayes estimators; p-value; Evidence measures

\section{Introduction}

Let $X$ be a normal random variable with distribution $N\left(\theta, \sigma^{2}\right)$, where $\theta$ is unknown and $\sigma^{2}$ is known. Let $\Theta_{0}$ be a subset of parameter space, which is an interval $\left[\theta_{0}, \theta_{1}\right]$ or contains only one point $\theta_{0}$. Consider the two sided hypothesis testing

$$
H_{0}: \theta \in \Theta_{0} \text { versus } H_{1}: \theta \notin \Theta_{0} .
$$


It is well known that there does not exist UMP tests for two-sided hypotheses testing. In this case, it is impossible to find a good one among all tests, then we focus on the class of unbiased tests instead of all tests. A test with power function $\beta(\theta)$ is unbiased if $\beta\left(\theta^{\prime}\right) \geq \beta\left(\theta^{\prime \prime}\right)$ for every $\theta^{\prime} \in \Theta_{0}^{c}$ and $\theta^{\prime \prime} \in \Theta_{0}$. An uniformly most powerful test (UMPU) is a UMP test within the class of unibased test. It is shown in (Lehmann (1997)) that UMPU test does exist for two-sided testing in exponential families. For $\Theta_{0}=\left[\theta_{0}, \theta_{1}\right]$, a level $\alpha$ UMPU test of (1) is of the form

$$
\phi(x)=\left\{\begin{array}{lll}
1 & \text { when } \quad x \leq c_{1} \text { or } \quad x \geq c_{2} \\
0 & \text { when } \quad c_{1}<x<c_{2}
\end{array}\right.
$$

where the c's are determined by

$$
E_{\theta_{0}} \phi(X)=E_{\theta_{1}} \phi(X)=\alpha
$$

When $\Theta_{0}$ contains only one point $\theta_{0}$, a level $\alpha$ UMPU test of (1) is of the form

$$
\phi(x)=\left\{\begin{array}{lll}
1 & \text { when } \quad x \leq c_{1} \quad \text { or } \quad x \geq c_{2} \\
0 & \text { when } \quad c_{1}<x<c_{2},
\end{array}\right.
$$

where the c's are determined by

$$
E_{\theta_{0}}[\phi(X)]=\alpha \quad \text { and } \quad E_{\theta_{0}}[X \phi(X)]=E_{\theta_{0}}(X) \alpha
$$

The set $\{x: \phi(x)=1\}$ is the rejection region of the test. If the observations belong to the rejection region, the null hypothesis is rejected. In this approach, the significance level $\alpha$ should be specified first. Another way of reporting the results of a test is to report the $\mathrm{p}$-value. The $\mathrm{p}$-value for a sample point $x$ is the smallest value of $\alpha$ for which the sample point will lead to rejection of $H_{0}$. Although the p-value is defined in terms of $\alpha$ levels, it is not a significance level. We usually use the p-values as a measure of data-based evidence against $H_{0}$. However, using only p-value as evidence against null hypothesis might lead to a wrong decision (see Berger and Wolpert (1988)). Berger and Sellke (1987) and 
Berger and Delampady (1987) also have other criticisms for p-values. Thus, it is essential to set up a criterion to evaluate p-values. Schaafsma, Tolboom and Van der Menlen (1989) and Hwang, Casella, Robert, Wells and Farrel (1992) use the risk

$$
E\left(r(X)-I\left(\theta \in \Theta_{0}\right)\right)^{2}
$$

to evaluate p-value, where

$$
I\left(\theta \in \Theta_{0}\right)= \begin{cases}1 & \text { if } \theta \in \Theta_{0} \\ 0 & \text { otherwise }\end{cases}
$$

and $r(x)$ is an estimator of $I\left(\theta \in \Theta_{0}\right)$. In a testing problem, we have to decide to reject or accept the null hypothesis. Actually, this problem can be interpreted as the problem of estimating $I\left(\theta \in \Theta_{0}\right)$. Hence a squared error risk of the form (4) is a sensible criterion to evaluate evidence measures against null hypothesis.

Under the criterion (4), Hwang et al (1992) show that for one-sided testing problem, p-values of UMP tests are admissible estimators of $I\left(\theta \in H_{0}\right)$ for some distributions and, however, for two-sided testing problem, p-values of UMPU tests are inadmissible for general distributions. Hence, for a two-sided testing problem, there exist other better estimators for $I\left(\theta \in H_{0}\right)$. Bayes estimators with respect to some proper priors are good candidates because they are admissible estimators. It reveals that Bayes estimators are good measures of evidence against $H_{0}$ from decision theoretic perspective. However, it is perhaps unfair to discuss this problem only from the decision theoretic framework because the merit of using p-value of UMPU test as a measure of evidence is, from testing viewpoint, that the test is a UMPU test. Thus, in this paper, we will examine the Bayes estimators of $I\left(\theta \in H_{0}\right)$ from testing pointview and demonstrate that Bayes estimator has the same advantage as p-value from testing aspect. First, a $\alpha$ level test derived from an estimator 
$r(x)$ of $I\left(\theta \in H_{0}\right)$ is of the form

$$
\phi(x)=\left\{\begin{array}{lll}
1 & \text { if } \quad r(x) \leq k \\
0 & \text { if } \quad r(x)>k
\end{array}\right.
$$

where $k$ is a positive constant between 0 and 1 such that $\max _{\theta \in \Theta_{0}} E_{\theta} \phi(x)=\alpha$. An estimator $r(x)$ of $I\left(\theta \in H_{0}\right)$ represents a confidence of $\theta$ belonging $\Theta_{0}$. Thus, a reasonable level $\alpha$ test derived from estimator $r(x)$ should be of the form (5). Consequently, if $r(x)$ in (5) is replaced by p-value of UMPU test, then $\phi(x)$ is a UMPU test, which is the advantage that people use the p-values as a measure of evidence against null hypothesis.

In this paper, the test derived from some Bayes estimators of $I\left(\theta \in \Theta_{0}\right)$ will be shown to be UMPU test. Thus, these Bayes estimators have the same merit as p-values from testing point of view, but it is superior to p-values from decision theoretical point of view. Combining these results, the Bayes estimators are strong competitors of p-values as measures of evidence against null hypothesis.

In this paper, the parameter space is assumed to be the natural parameter space $(-\infty, \infty)$. When the parameter space is restricted to some subset of natural parameter space, Woodroofe and Wang (2001) show that p-value of UMP test is inadmissible from decision theoretical aspect for one-sided testing problem of Poisson distribution and purposed a Bayes estimator to be a modified p-value. Moreover, for simple hypothesis versus simple hypothesis case, Wang (2004) demonstrated that some Bayes estimator is better than the p-value of most powerful test, which reveals that Bayes estimator is a good substitute of p-value when the parameter space is restricted. In this paper, Bayes estimators are demonstrated to be a good measure of evidence for twosided testing when the parameter space is natural parameter space. Hence, Bayes estimators are recommended to be a substitute of p-value as a measure of evidence against null hypothesis. 


\section{The case of $\Theta_{0}=\left[\theta_{0}, \theta_{1}\right]$}

In this section, we consider the case of $\Theta_{0}=\left[\theta_{0}, \theta_{1}\right]$. The other case that $\Theta_{0}$ contains only one point is discussed in next section. For $\Theta_{0}=\left[\theta_{0}, \theta_{1}\right]$, the test derived from the Bayes estimator $\eta(x)$ of $I\left(\theta \in \Theta_{0}\right)$ with respect to uniform prior $U\left(2 \theta_{0}-\theta_{1}, 2 \theta_{1}-\theta_{0}\right)$ will be shown to be a UMPU test. A main mathematics technique used in this section is solving equations of cubic polynomials.

Lemma 1. (Jacobson (1985), p.260, exercise 2) Let $f(x)=x^{3}-p x^{2}+q x-r$ be a cubic polynomial, where $p, q$ and $r$ are three real numbers $(p, q$ and $r$ $\in \Re)$. Then $f(x)$ has three real distinct roots if

$$
\triangle(f)=p^{2} q^{2}-4 p^{3} r+18 p q r-4 q^{3}-27 r^{2}>0
$$

where $\triangle(f)$ is called the discriminant of $f(x)$.

Lemma 2. Let

$$
\eta(x)=\int_{\theta_{0}}^{\theta_{1}} g(\theta-x) d \theta / \int_{2 \theta_{0}-\theta_{1}}^{2 \theta_{1}-\theta_{0}} g(\theta-x) d \theta
$$

where $g(x-\theta)=e^{-(x-\theta)^{2} / 2} / \sqrt{2 \pi}$. For every $x$, there exist a $\omega$ such that $g\left(x-\theta_{0}\right)=g\left(\omega-\theta_{1}\right)$ and $\eta(x)=\eta(\omega)$.

Proof. For any $x$, the only two solutions of $\omega$ such that

$$
g\left(x-\theta_{0}\right)=g\left(\omega-\theta_{1}\right)
$$

are $\omega=x-\theta_{0}+\theta_{1}$ and $\omega=-x+\theta_{0}+\theta_{1}$. Note that $\eta(x)$ and $\eta(\omega)$ can be rewritten as

$$
\frac{\int_{\theta_{0}-x}^{\theta_{1}-x} e^{-\frac{t^{2}}{2}} d t}{\int_{2 \theta_{0}-\theta_{1}-x}^{2 \theta_{1}-\theta_{0}-x} e^{-\frac{t^{2}}{2}} d t}
$$

and

$$
\frac{\int_{\theta_{0}-\omega}^{\theta_{1}-\omega} e^{-\frac{t^{2}}{2}} d t}{\int_{2 \theta_{0}-\theta_{1}-\omega}^{2 \theta_{1}-\theta_{0}-\omega} e^{-\frac{t^{2}}{2}} d t}
$$


If $w$ is replaced with $-x+\theta_{0}+\theta_{1}$ in (7), then by the fact that $e^{-t^{2} / 2}$ is symmetric to zero, we have $\eta(\omega)=\eta(x)$. Thus, $\omega$ can be chosen as $-x+\theta_{0}+\theta_{1}$.

Lemma 3. Let

$d(x)=(1-k) \int_{\theta_{0}}^{\theta_{1}} e^{-(\theta-x)^{2} / 2} d \theta-k \int_{2 \theta_{0}-\theta_{1}}^{\theta_{0}} e^{-(\theta-x)^{2} / 2} d \theta-k \int_{\theta_{1}}^{2 \theta_{1}-\theta_{0}} e^{-(\theta-x)^{2} / 2} d \theta$.

(i) When $k<1 / 3$, d $(x)$ has a local maximum at $\left(\theta_{0}+\theta_{1}\right) / 2$ for $\theta_{1}>\theta_{0}$.

(ii) When $k \geq 1 / 3$, $d(x)$ has a local maximum at $\left(\theta_{0}+\theta_{1}\right) / 2$ for $\theta_{1}-\theta_{0} \geq$ $\sqrt{\ln 3 k}$.

Proof. The first and second derivates of $d(x)$ with respect to $x$ are

$$
\begin{aligned}
& -(1-k)\left[e^{-\left(\theta_{1}-x\right)^{2} / 2}-e^{-\left(\theta_{0}-x\right)^{2} / 2}\right]+k\left[e^{-\left(\theta_{0}-x\right)^{2} / 2}-e^{-\left(2 \theta_{0}-\theta_{1}-x\right)^{2} / 2}\right] \\
& +k\left[e^{-\left(2 \theta_{1}-\theta_{0}-x\right)^{2} / 2}-e^{-\left(\theta_{1}-x\right)^{2} / 2}\right]
\end{aligned}
$$

and

$$
\begin{aligned}
& e^{-\left(\theta_{1}-x\right)^{2} / 2}\left(x-\theta_{1}\right)-e^{-\left(\theta_{0}-x\right)^{2} / 2}\left(x-\theta_{0}\right)+k e^{-\left(2 \theta_{0}-\theta_{1}-x\right)^{2} / 2}\left(x-2 \theta_{0}+\theta_{1}\right) \\
& -k e^{-\left(2 \theta_{1}-\theta_{0}-x\right)^{2} / 2}\left(x-2 \theta_{1}+\theta_{0}\right) .
\end{aligned}
$$

If $x$ is replaced with $\left(\theta_{0}+\theta_{1}\right) / 2$ in (10), then (10) is equal to

$$
\left(\theta_{1}-\theta_{0}\right) e^{-9\left(\theta_{1}-\theta_{0}\right)^{2} / 8}\left[3 k-e^{\left(\theta_{1}-\theta_{0}\right)^{2}}\right]
$$

Since $\left(\theta_{0}+\theta_{1}\right) / 2$ is a root of $\frac{\partial}{\partial x} d(x)$ and (10) is the second derivate of $d(x)$, if we can show that $(11)$ is less than zero, then $d(x)$ has a local maximum at $\left(\theta_{0}+\theta_{1}\right) / 2$. When $k<1 / 3$, (11) is less than zero. When $k \geq 1 / 3$, (11) is less than zero if and only if $\theta_{1}-\theta_{0} \geq \sqrt{\ln 3 k}$. Hence the proof is completed. 
Lemma 4. Let $\xi(x)=F(x / 2)-F(-x / 2)+k F(-3 x / 2)-k F(3 x / 2), x>0$, where $F(\cdot)$ is the cumulative function of standard normal distribution $N(0,1)$. Then when $k<1 / 3, \xi(x)$ is positive and, when $k \geq 1 / 3, \xi(x)$ is positive if and only if $x>\nu$, where $\nu$ satisfies $\xi(x)=0$.

Proof.

$$
\frac{\partial}{\partial x} \xi(x)=e^{-x^{2} / 8}\left(1-3 k e^{-x^{2}}\right)
$$

(i) When $k<1 / 3, \frac{\partial}{\partial x} \xi(x)>0$ for $-\infty<x<\infty$. And $\xi(x)=0$ when $x=0$ and $\xi(x)=1-k$ when $x=\infty$. The figure of $\xi(x)$ to $x$ is Figure 1 . Note that $\xi(x)$ is positive when $x$ is greater than zero.

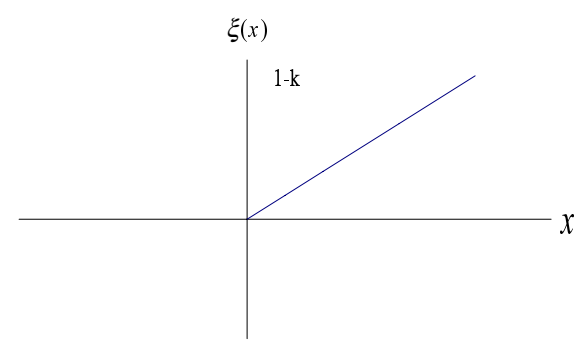

Figure 1: Plot of $\xi(x)$

(ii) When $k \geq 1 / 3, \frac{\partial}{\partial x} \xi(x)=0$ if and only if $x=\infty$ and $\sqrt{\ln 3 k}$. By the fact that $\xi(x)=1-k$ at $x=\infty, \xi(0)=0, \frac{\partial}{\partial x} \xi(x)<0$ when $0<x<\sqrt{\ln 3 k}$ and $\frac{\partial}{\partial x} \xi(x)>0$ when $x>\sqrt{\ln 3 k}$. It leads to $\xi(\sqrt{\ln 3 k})<0$ and the figure of $\xi(x)$ to $x$ is Figure 2. Note that $\nu$ is greater than $\sqrt{\ln 3 k}$.

Combining Lemma 1-4, we have the main result in Theorem 1. 


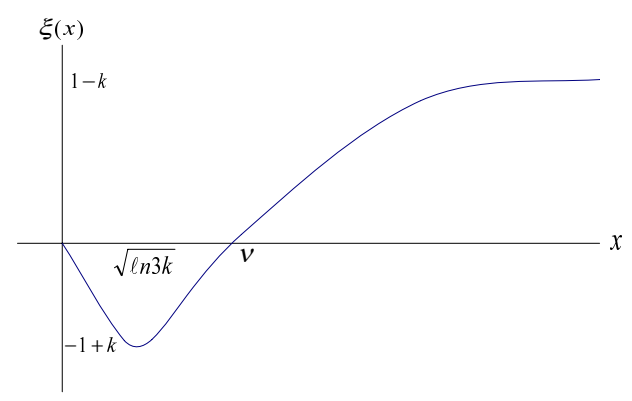

Figure 2: Plot of $\xi(x)$

Theorem 1. Let $X$ be a normal random variable with distribution $N\left(\theta, \sigma^{2}\right)$, where $\sigma^{2}$ is known. In hypotheses testing (1), consider the test

$$
\phi(x)=\left\{\begin{array}{lll}
1 & \text { if } & \eta(x) \leq k \\
0 & \text { if } & \eta(x)>k
\end{array}\right.
$$

where $\eta(x)$ is a Bayes estimator of $I\left(\theta \in H_{0}\right)$ with respect to uniform prior $U\left(2 \theta_{0}-\theta_{1}, 2 \theta_{1}-\theta_{0}\right)$ and $k$ is a positive constant such that $E_{\theta_{0}} \phi(X)=\alpha$, then

(i) When $k \leq 1 / 3, \phi(x)$ is $\alpha$ - level UMPU test for $\theta_{0}$ and $\theta_{1}$ satisfying $\theta_{0}<\theta_{1}$.

(ii) When $k>1 / 3, \phi(x)$ is a $\alpha$-level UMPU test for $\theta_{0}$ and $\theta_{1}$ satisfying $\theta_{1}-\theta_{0}>\nu$, where $\nu$ is defined in Lemma 4.

Proof. Without loss of generality, we assume that $\sigma^{2}=1$. The rejection region of null hypothesis is

$$
\left\{x: \frac{\int_{\theta_{0}}^{\theta_{1}} f(\theta-x) d \theta}{\int_{2 \theta_{0}-\theta_{1}}^{22_{1}-\theta_{0}} f(\theta-x) d \theta} \leq k\right\}
$$

which is equal to $\{x: d(x) \leq 0\}$, where $d(x)$ is defined in (8). The first derivative of $d(x)$ is (9).

Let $m=\theta_{1}-\theta_{0}$ and $y=e^{m\left(x-\theta_{0}\right)}$. Then, (9) can be rewritten as

$$
k e^{\left(-\frac{\left(x-\theta_{0}\right)^{2}}{2}-2 m^{2}\right)}\left[y^{3}-e^{3 m^{2} / 2} y^{2} / k+e^{2 m^{2}} y / k-e^{3 m^{2} / 2}\right] .
$$


Let

$$
f(y)=y^{3}-\left(e^{3 m^{2} / 2} / k\right) y^{2}+\left(e^{2 m^{2}} / k\right) y-e^{3 m^{2} / 2} .
$$

By Lemma 1, $\triangle(f(y))=e^{3 m^{2}}\left[e^{4 m^{2}}-8 k e^{3 m^{2}}-27 k^{4}\right] / k^{4}$, which is equal to

$$
e^{3 m^{2}}\left(e^{m^{2}}-3 k\right)^{3}\left(e^{m^{2}}+k\right) / k^{4} .
$$

Note that $k$ is greater than zero, thus, (15) is greater than zero if $e^{m^{2}}>3 k$. Then we will proceed to the proof by consider two cases : (i) $k<1 / 3$, (ii) $k \geq 1 / 3$. First, we consider the case (i). Since $k<1 / 3$, which leads to $e^{m^{2}}>3 k,(15)$ is greater than zero for all $m$. Thus the three degree polynomial in (14) has three distinct real roots. Assume that the three roots are $y_{1}, y_{2}$ and $y_{3} \quad\left(y_{1}<y_{2}<y_{3}\right)$. Hence $\frac{\partial}{\partial x} d(x)$ is less than zero when $y<y_{1}$ or $y_{2}<y<y_{3}$ and $\frac{\partial}{\partial x} d(x)$ is greater than zero when $y_{1}<y<y_{2}$ or $y>y_{3}$. It means that $d(x)$ is decreasing when $y<y_{1}$ and $y_{2}<y<y_{3}$ and $d(x)$ is increasing when $y_{1}<y<y_{2}$ and $y>y_{3}$. Moreover, $d(x)$ can be rewritten as

$$
(1-k) \int_{\theta_{0}-x}^{\theta_{1}-x} e^{-t^{2} / 2} d t-k \int_{2 \theta_{0}-\theta_{1}-x}^{\theta_{0}-x} e^{-t^{2} / 2} d t-k \int_{\theta_{1}-x}^{2 \theta_{1}-\theta_{0}-x} e^{-t^{2} / 2} d t .
$$

When $x$ goes to infinity and minus infinity, (16) goes to zero. Thus, combining the above arguments, $d(x)$ has one local maximum and two local minimum. Note that $\left(\theta_{0}+\theta_{1}\right) / 2$ is one root of $(9)$. By Lemma $3, d(x)$ has a local maximum at $x=\left(\theta_{0}+\theta_{1}\right) / 2 . d\left(\left(\theta_{0}+\theta_{1}\right) / 2\right)$ is equal to $F\left(\left(\theta_{1}-\theta_{0}\right) / 2\right)-$ $F\left(\left(\theta_{0}-\theta_{1}\right) / 2\right)+k F\left(3\left(\theta_{0}-\theta_{1}\right) / 2\right)-k F\left(3\left(\theta_{1}-\theta_{0}\right) / 2\right)$, where $F(\cdot)$ is the cumulative function of standard normal distribution. By Lemma 4 , when $k<1 / 3$, $d\left(\left(\theta_{0}+\theta_{1}\right) / 2\right)$ is always greater than zero for all $\theta_{0}$ and $\theta_{1}$ satisfying $\theta_{1}-\theta_{0}>0$. Thus, the figure of $d(x)$ to $y$ is Figure 3 .

By Figure 3, it leads to that the region $\{x: d(x) \leq 0\}$ is

$$
\left\{x: y<\mu_{1}^{*} \quad \text { or } \quad y>\mu_{2}^{*}\right\}
$$

where $\mu_{1}^{*}$ and $\mu_{2}^{*}$ are two values such that $d\left(\mu_{1}^{*}\right)=d\left(\mu_{2}^{*}\right)=0$. (see Figure 3 ). Let $c_{1}=\ln \mu_{1}^{*} / m+\theta_{0}$ and $c_{2}=\ln \mu_{2}^{*} / m+\theta_{0}$. Then by the definition of $y,(17)$ 


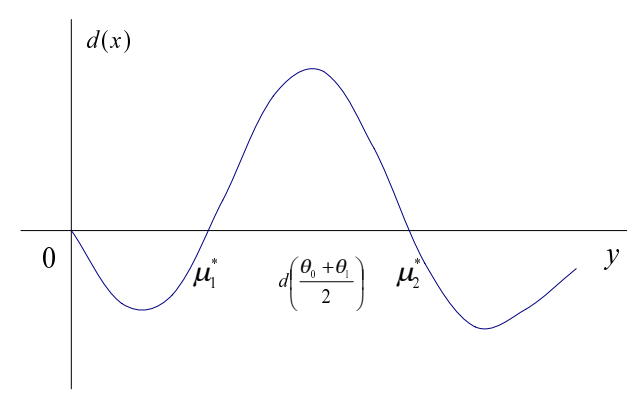

Figure 3: Plot of $d(x)$

is equivalent to

$$
\left\{x: x<c_{1} \quad \text { or } \quad x>c_{2}\right\}
$$

Note that $k$ is chosen to be satisfied $E_{\theta_{0}} \phi(X)=\alpha$. Hence by the form of the rejection region (18) which is the same as the form of UMPU test and $E_{\theta_{0}} \phi(X)=\alpha$, the proof of case (i) will be completed if we prove $E_{\theta_{1}} \phi(X)=\alpha$. ¿From Lemma 2 , for every $x$, there exists a $\omega$ such that $e^{-\left(x-\theta_{0}\right)^{2} / 2}=e^{-\left(\omega-\theta_{1}\right)^{2} / 2}$ and $\eta(x)=\eta(\omega)$. Then it will lead to $E_{\theta_{1}} \phi(X)=\alpha$.

Now we will consider case (ii) $(k \geq 1 / 3)$. When $k \geq 1 / 3,(15)$ is greater than zero only when $m=\theta_{1}-\theta_{0}>\sqrt{\ln 3 k}$. And by Lemma $4, d\left(\left(\theta_{0}+\theta_{1}\right) / 2\right)$ is greater than zero only when $\theta_{1}-\theta_{0}>\nu$ and in Lemma 4 , we know that $\nu>\sqrt{\ln 3 k}$. Thus, when $\theta_{1}-\theta_{0}>\nu$, by a similar argument as in case (i), the proof is completed.

The value of $\nu$ in Theorem 1 is defined in Lemma 4 and Table 1 is the value of $\nu$ corresponding to $k \geq 1 / 3$ by numerical calculation.

Table 1

\begin{tabular}{|c|c|c|c|c|c|c|c|c|c|c|c|c|}
\hline $\mathrm{k}$ & 0.35 & 0.4 & 0.45 & 0.5 & 0.55 & 0.6 & 0.65 & 0.7 & 0.75 & 0.8 & 0.85 & 0.9 \\
\hline$\nu$ & 0.39 & 0.78 & 1.04 & 1.26 & 1.46 & 1.66 & 1.86 & 2.07 & 2.30 & 2.56 & 2.88 & 3.29 \\
\hline
\end{tabular}




\section{The case of $\Theta_{0}=\theta_{0}$}

If the null hypothesis sample space contains only one point, the likelihood ratio test is a common tool for testing two-sided hypotheses. For the normal distribution, the likelihood ratio test and UMPU test are the same. Theorem 2 and Theorem 3 are the main results of this section. Without loss of generality, $\sigma^{2}$ is assumed to be 1 in Theorem 2 and Theorem 3.

Theorem 2. Let $X_{1}, \cdots, X_{n}$ be iid according to $N(\theta, 1)$. For testing (1), assume that the null parameter space $\Theta_{0}$ contains only one point $\theta_{0}$, then the test

$$
\phi(x)= \begin{cases}1 & \text { if } \quad \eta_{1}(x) \leq k \\ 0 & \text { if } \quad \eta_{1}(x)>k\end{cases}
$$

is a level $\alpha$ UMPU test, which is also a likelihood ratio test, where

$\eta_{1}(x)=m e^{-\sum_{i=1}^{n}\left(x_{i}-\theta_{0}\right)^{2} / 2} /\left(e^{-\sum_{i=1}^{n}\left(x_{i}-t_{1}\right)^{2} / 2}+m e^{-\sum_{i=1}^{n}\left(x_{i}-\theta_{0}\right)^{2} / 2}+e^{-\sum_{i=1}^{n}\left(x_{i}-t_{2}\right)^{2} / 2}\right), t_{1}<$

$\theta_{0}<t_{2}, \theta_{0}-t_{1}=r, t_{2}-t_{1}=2 r, k=m e^{c n r+n r^{2} / 2} /\left(1+e^{2 c n r}+m e^{c n r+n r^{2} / 2}\right)$, $m(0<m<\infty)$ and $r(0<r<\infty)$ are some constants and $c$ is the upper $\alpha / 2$ quantile of $N(0,1)$.

Proof. The rejection region of $\phi(x)$ is equivalent to

$$
\left\{x: m(1-k) e^{-\sum_{i=1}^{n}\left(x_{i}-\theta_{0}\right)^{2} / 2}-k e^{-\sum_{i=1}^{n}\left(x_{i}-t_{1}\right)^{2} / 2}-k e^{-\sum_{i=1}^{n}\left(x_{i}-t_{2}\right)^{2} / 2} \leq 0\right\} .
$$

By dividing $e^{-\sum_{i=1}^{n}\left(x_{i}-\theta_{0}\right)^{2} / 2}$ in both side of (19) and let $y=e^{n\left(\bar{x}-\theta_{0}\right) r}$, (19) is equivalent to

$$
\{y: h(y) \geq 0\}
$$

where

$$
h(y)=k e^{-n r^{2} / 2}\left[y^{2}-m(1-k) e^{n r^{2} / 2} y / k+1\right] .
$$

Thus, $\frac{\partial}{\partial y} h(y)=k e^{-n r^{2} / 2}\left[2 y-m(1-k) e^{n r^{2} / 2} / k\right]$ and let $y_{0}$ be the root of $\frac{\partial}{\partial y} h(y)=0$. Note that $\frac{\partial^{2} h(y)}{\partial^{2} y}=2 k e^{-n r^{2} / 2}$, which is always greater than zero. 
Hence $h\left(y_{0}\right)$ has a local minimum at $y_{0}$ and $h\left(y_{0}\right)=1-e^{n r^{2}}(1-k)^{2} m^{2} /\left(4 k^{2}\right)$. By the definition of $y$, we need only consider $h(y)$ with respect to positive part of $y$. By the facts that $h(0)>0, h(y)>0$ when $y$ goes to infinity, $\frac{\partial}{\partial y} h(y)<0$ when $0<y<y_{0}$ and $\frac{\partial}{\partial y} h(y)>0$ when $y>y_{0}$. Moreover, since $k=m e^{c n r+n r^{2} / 2} /\left(1+e^{2 c n r}+m e^{c n r+n r^{2} / 2}\right)$ satisfying $e^{n r^{2}} m^{2}>4 k^{2} /(1-k)^{2}$, which leads to $h\left(y_{0}\right)<0$. Therefore, the figure of $h(y)$ to $y$ is Figure 4 .

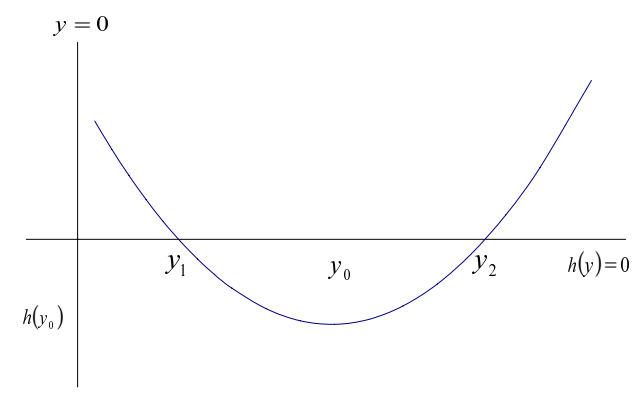

Figure 4: Plot of $h(y)$

The two roots of $h(y)=0$ are

$$
y_{1}=\left(e^{n r^{2} / 2}(1-k) m-\sqrt{-4 k^{2}+e^{n r^{2}}(1-k)^{2} m^{2}}\right) / 2 k .
$$

and

$$
y_{2}=\left(e^{n r^{2} / 2}(1-k) m+\sqrt{-4 k^{2}+e^{n r^{2}}(1-k)^{2} m^{2}}\right) / 2 k .
$$

By (20), the rejection region of $\phi(x)$ is $\left\{y: y<y_{1}\right.$ and $\left.y>y_{2}\right\}$. By the definition of $y,(\bar{x}-\theta)=(\ln y) / n r$, which leads that the rejection region is $\left\{\bar{x}:\left(\bar{x}-\theta_{0}\right)<\left(\ln y_{1}\right) / n r\right.$ and $\left.\left(\bar{x}-\theta_{0}\right)>\left(\ln y_{2}\right) / n r\right\}$. By straightforward calculation, $y_{1} y_{2}=1$, which means that $\phi(x)$ is a likelihood ratio test because of $\ln y_{1} / n r=-\ln y_{2} / n r$. By Casella and Berger (1990), the rejection region of a UMPU test for a normal distribution is also the same as this rejection region. Hence $\phi(x)$ is also a UMPU test. Moreover, according to $\alpha$-level likelihood ratio test, $\ln y_{2} / n^{2} r=-\ln y_{1} / n^{2} r=c$, where $c$ 
is a upper quantile of $N(0,1)$. Then, by solving the above two equations, $k=e^{c n r+n r^{2} / 2} m /\left(1+e^{2 c n r}+e^{c n r+n r^{2} / 2} m\right)$.

Lemma 5. The 4th degree polynomial equation $y^{4}+e^{3 n r^{2} / 2} y^{3}-m(1-k) / k e^{2 n r^{2}} y^{2}+$ $e^{3 n r^{2} / 2} y+1=0$ has 4 real roots at $r_{1}, r_{2}, r_{3}$ and $r_{4}$ if $n r^{2}$ is large, where

$$
\begin{gathered}
r_{1}=-\frac{1}{4} e^{3 n r^{2} / 2}-A-\frac{1}{2}(B-C)^{\frac{1}{2}}, r_{2}=-\frac{1}{4} e^{3 n r^{2} / 2}-A+\frac{1}{2}(B-C)^{\frac{1}{2}} \\
r_{3}=-\frac{1}{4} e^{3 n r^{2} / 2}+A-\frac{1}{2}(B+C)^{\frac{1}{2}}, r_{4}=-\frac{1}{4} e^{3 n r^{2} / 2}+A+\frac{1}{2}(B+C)^{\frac{1}{2}}, \\
A=\frac{1}{2}\left(2+\frac{1}{4} e^{3 n r^{2}}-e^{2 n r^{2}}(-1+k) m / k\right)^{\frac{1}{2}}, \\
B=-2+\frac{1}{2} e^{3 n r^{2}}-e^{2 n r^{2}}(-1+k) m / k
\end{gathered}
$$

and

$$
C=\frac{-8 e^{3 n r^{2} / 2}-e^{9 n r^{2} / 2}+4 e^{7 n r^{2} / 2}(-1+k) m / k}{4 \sqrt{2+\frac{1}{4} e^{3 n r^{2}}-e^{2 n r^{2}}(-1+k) m / k}} .
$$

Proof. By straightforward calculation, $\left(y-r_{1}\right)\left(y-r_{2}\right)\left(y-r_{3}\right)\left(y-r_{4}\right)$ is equal to the lefthand side of the equation. The method of solving 4 th degree of polynomial referres to Zwillinger, Krantz and Rosen (1996).

Theorem 3. Let $X_{1}, \cdots, X_{n}$ be iid according to $N(\theta, 1)$. For testing (1), assumed that the null parameter space contains only one point $\theta_{0}$, then the test

$$
\phi(X)=\left\{\begin{array}{lll}
1 & \text { if } \quad \eta_{2}(X) \leq k \\
0 & \text { if } \quad \eta_{2}(X)>k
\end{array}\right.
$$

is a level a UMPU test, which is also a likelihood ratio test, where $\eta_{2}(x)=$ $m e^{-\left(x-\theta_{0}\right)^{2} / 2} /\left[\left(\sum_{j=1}^{4} e^{-\left(x-t_{j}\right)^{2} / 2}\right)+m e^{-\left(x-\theta_{0}\right)^{2} / 2}\right], t_{1}<t_{2}<\theta_{0}<t_{3}<t_{4}, t_{2}-$ $t_{1}=\theta_{0}-t_{2}=t_{3}-\theta_{0}=t_{4}-t_{3}=r, k=e^{2 n r(c+4 r)} m /\left(e^{6 n r^{2}}+e^{2 n r(2 c+3 r)}+\right.$ $\left.e^{3 n r(2 c+5 r) / 2}+e^{c n r+15 n r^{2} / 2}+e^{2 n r(c+4 r)} m\right), m$ and $r$ are some constants and $c$ is the upper $\alpha / 2$ quantile of $N(0,1)$. 
Proof. The rejection region of $\phi(X)$ is

$$
\left\{X: m e^{-\sum_{i=1}^{n}\left(x_{i}-\theta_{0}\right)^{2} / 2} /\left(m e^{-\sum_{i=1}^{n}\left(x_{i}-\theta_{0}\right)^{2} / 2}+\sum_{j=1}^{4} e^{-\sum_{i=1}^{n}\left(x_{i}-t_{j}\right)^{2} / 2}\right) \leq k\right\},
$$

which is equivalent to

$$
\left\{X: m(1-k) e^{-\sum_{i=1}^{n}\left(x_{i}-\theta_{0}\right)^{2} / 2}-k \sum_{j=1}^{4} e^{-\sum_{i=1}^{n}\left(x_{i}-\theta_{j}\right)^{2} / 2} \leq 0\right\} .
$$

By dividing $e^{-\sum_{i=1}^{n}\left(x_{i}-\theta_{0}\right)^{2} / 2}$ in both side, $(21)$ is equal to

$$
\left\{X: m(1-k)-k\left(\sum_{j=1}^{4} e^{n\left(\bar{x}-\theta_{0}\right)\left(t_{j}-\theta_{0}\right)-n\left(\theta_{0}-t_{j}\right)^{2} / 2}\right) \leq 0\right\} .
$$

Let $y=e^{n\left(\bar{x}-\theta_{0}\right) r}$. By the fact that $t_{2}-t_{1}=\theta_{0}-t_{2}=t_{3}-\theta_{0}=t_{4}-t_{3}=r$, (22) can be rewritten as

$$
\left\{-m(1-k)+k\left(y^{-2} e^{-2 n r^{2}}+y^{-1} e^{-n r^{2} / 2}+y e^{-n r^{2} / 2}+y^{2} e^{-2 n r^{2}}\right) \geq 0\right\},
$$

which can also be written as

$$
\left\{X: k y^{-2} e^{-2 n r^{2}}\left(y^{4}+e^{3 n r^{2} / 2} y^{3}-m(1-k) / k e^{2 n r^{2}} y^{2}+e^{3 n r^{2} / 2} y+1\right) \geq 0\right\}
$$

Let

$$
h(y)=y^{4}+e^{3 n r^{2} / 2} y^{3}-m(1-k) / k e^{2 n r^{2}} y^{2}+e^{3 n r^{2} / 2} y+1
$$

$\frac{\partial}{\partial y} h(y)=4 y^{3}+3 e^{3 n r^{2} / 2} y^{2}-2 m(1-k) / k e^{2 n r^{2}} y+e^{3 n r^{2} / 2}$. By Lemma 1, let $p=-3 e^{3 n r^{2} / 2} / 4, q=-2 m(1-k) e^{2 n r^{2}} /(4 k)$ and $r=-e^{3 n r^{2} / 2} / 4$, then we have the discriminant

$$
\begin{aligned}
\Delta\left(\frac{\partial}{\partial y} h(y)\right)= & e^{3 n r^{2}}\left[-108+108 e^{2 n r^{2}}(-1+k) m / k+9 e^{4 n r^{2}}(-1+k)^{2} m^{2} / k^{2}+\right. \\
& \left.e^{3 n r^{2}}\left(-27-(-1+k)^{3} m^{3} / k^{3}\right)\right] / 64
\end{aligned}
$$

When $n r^{2}$ is large enough, the leading term of $\Delta\left(\frac{\partial}{\partial y} h(y)\right)$ is $9 e^{7 n r^{2}}(1-k)^{2} m^{2}$, which is positive. By Lemma 1, it leads that $\frac{\partial}{\partial y} h(y)$ has three distinct roots, say $y_{0}, y_{1}, y_{2}$. Note that $h(y)>0$ if $y$ is zero and $h(y)>0$ if $y$ goes to infinity. 
Moreover, by Lemma 5 , the equation $h(y)=0$ has four roots $r_{1}, r_{2}, r_{3}$ and $r_{4}$. By straightforward calculation, $r_{1} \times r_{2}=1$ and $r_{3} \times r_{4}=1$. Note that it is obvious that $r_{1}$ is negative and $r_{4}$ is positive if $n$ is large enough because the terms involving $e^{3 n r^{2} / 2}$ are leading terms in these roots. Since $r_{1} \times r_{2}$ is positive and $r_{3} \times r_{4}$ is also positive, thus, $r_{2}$ is negative and $r_{3}$ is positive. Combining the above arguments, the figure of $h(y)$ with respect to $y$ is figure 5 .

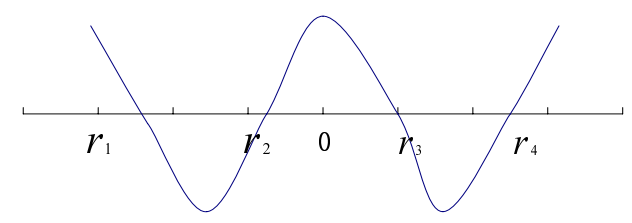

Figure 5: Plot of $h(y)$

By the defintion of $y, y$ is always positive. Thus, the figure of $h(y)$ with respect to $y>0$ is

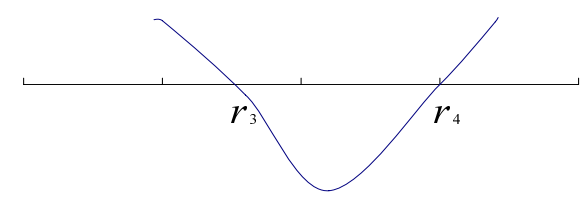

Figure 6: Plot of $h(y)$

By (23), the rejection region is $\left\{y: 0<y<r_{3} \quad\right.$ or $\left.y>r_{4}\right\}$, which is corresponding to

$$
\left\{x: \bar{x}-\theta_{0}<\ln r_{3} / n r \quad \text { or } \quad \bar{x}-\theta_{0}>\ln r_{4} / n r\right\} .
$$


Note that $r_{3} \times r_{4}=1$. Thus (25) is equal to $\left\{x: \bar{x}-\theta_{0}<-\ln r_{4} / n r\right.$ or $\bar{x}-$ $\theta_{0}>$

$\left.\ln r_{4} / n r\right\}$, which is a UMPU test and also a likelihood ratio test. Moreover, $-\ln r_{3} / n r=\ln r_{4} / n r=c$, where $c$ is a upper $\alpha / 2$ quantile of $N(0,1)$. By solving the above two equations, we have $k=e^{2 n r(c+4 r)} m /\left(e^{6 n r^{2}}+e^{2 n r(2 c+3 r)}+\right.$ $\left.e^{3 n r(2 c+5 r) / 2}+e^{c n r+15 n r^{2} / 2}+e^{2 n r(c+4 r)} m\right)$.

The priors of the Bayes estimators in Theorem 2 and Theorem 3 are $I_{\left(\theta=t_{1}, t_{2}\right)}$ and $I_{\left(\theta=t_{1}, t_{2}, t_{3}, t_{4}\right)}$ respectively, where

$$
I_{\left(\theta=t_{1}, \cdots, t_{g}\right)}= \begin{cases}1 & \text { if } \theta=t_{1}, t_{2}, \cdots t_{g} \\ 0 & \text { otherwise }\end{cases}
$$

These points $\left\{t_{i}, i=1, \cdots, g\right\}$ are suggested to be some possible values of $\theta$ if we have information of $\theta$. In Theorem 2 and Theorem $3, g$ is chosen to be 2 or 4. If $g$ is chosen to be greater than 4 , then it is difficult to have general results because by Abel's theorem, one of the principal results of Galois Theory, there are no general solutions for solving $g$ th degree polynomial equations for $g \geq 5$ (Fraleigh (1982), Herstein (1975)). But, although it is impossible to establish general results for $g \geq 5$, we will investigate it from numerical study.

Proposition 1. If $\eta_{1}(x)$ in Theorem 2 is replaced with $m e^{-\sum_{i=1}^{n}\left(x_{i}-\theta_{0}\right)^{2} / 2} /$ $\left(\sum_{j=1}^{g} e^{-\sum_{i=1}^{n}\left(x_{i}-t_{j}\right)^{2} / 2}+m e^{-\sum_{x=1}^{n}\left(x_{i}-\theta_{0}\right)^{2} / 2}\right)$, where $g$ is an even positive integer, $t_{i}-\theta_{0}=(i-(g / 2+1)) r$ for $i \leq g / 2$ and $t_{i}-\theta_{0}=(i-g / 2) r$ for $i>g / 2$. Then the rejection region $\left\{x: \eta_{1}(x) \leq k\right\}$ is equal to $\left\{y:-y^{g / 2} m(1-k) / k+\right.$ $\left.\sum_{j=1}^{g / 2}\left(y^{g / 2-j} e^{-n r^{2} j^{2} / 2}+y^{g / 2+j} e^{-n r^{2} j^{2} / 2}\right)>0\right\}$, where $y=e^{n\left(\bar{x}-\theta_{0}\right) r}$.

The proof of Proposition 1 is by straightforward calculation. For $g=6$, the rejection region is $\left\{y: y^{6}+e^{5 n r^{2} / 2} y^{5}+e^{4 n r^{2}} y^{4}-m(1-k) / k e^{9 n r^{2} / 2} y^{3}+\right.$ $\left.e^{4 n r^{2}} y^{2}+e^{5 n r^{2} / 2} y+1 \geq 0\right\}$. From numerical calculation, the equation $y^{6}+$ $e^{5 n r^{2} / 2} y^{5}+e^{4 n r^{2}} y^{4}-m(1-k) / k e^{9 n r^{2} / 2} y^{3}+e^{4 n r^{2}} y^{2}+e^{5 n r^{2} / 2} y+1=0$ only has two positive roots for most values of $k, m, n$, and $r$. For example, $k=0.1$, 
$m=1$ and $n r^{2}=10$, the two positive roots of the equation are 0.000749 and 1335.17. Then by a similar argument as in Theorem 3, the tests derived from most Bayes estimators of the form $\eta_{1}(x)$ corresponding to $g=6$ are UMPU tests. For $g=8$, from numerical calculation and a similar argument as above, the tests derived from $\eta_{1}(x)$ corresponding to $g=8$ for most values of $k, m, n$ and $r$ are UMPU tests. According to the numerical analysis of $g=6$ and 8 , it is very possible that the tests derived from $\eta_{1}(x)$ corresponding to $g>8$ are UMPU tests.

\section{Simulation results}

In this section, the mean squared error of the proposed Bayes estimators and $\mathrm{p}$-value are compared for the case of $\Theta_{0}=\theta_{0}$. The mean squared error of an estimator $r(X)$ of $I\left(\theta \in \Theta_{0}\right)$ is $E\left(r(X)-I\left(\theta \in \Theta_{0}\right)\right)^{2}$. Let MSE 1, MSE 2 and MSE 3 denote the mean squared errors of $\eta_{1}(x), \eta_{2}(x)$ and p-value respectively. 
Table 2: The table is mean squared errors of three estimators of $I(\theta=0)$ when $m=0.4, r=1$ and $n=5$. The simulation is based on 3000 replicates.

\begin{tabular}{|c|c|c|c|}
\hline$\theta$ & MSE 1 & MSE 2 & MSE 3 \\
\hline-2 & 0.0002420 & 0.0002331 & 0.0001998 \\
\hline-1 & 0.0429601 & 0.0427160 & 0.0492836 \\
\hline 0 & 0.3520266 & 0.3532986 & 0.3404814 \\
\hline 1 & 0.0391925 & 0.0389499 & 0.0448714 \\
\hline 2 & 0.0002121 & 0.0002037 & 0.00017611 \\
\hline
\end{tabular}

Table 3: The table is mean squared errors of three estimators of $I(\theta=0)$ when $m=1.5, r=1$ and $n=5$. The simulation is based on 3000 replicates.

\begin{tabular}{|c|c|c|c|}
\hline$\theta$ & MSE 1 & MSE 2 & MSE 3 \\
\hline-2 & 0.0009975 & 0.0009100 & 0.0000988 \\
\hline-1 & 0.1213554 & 0.120094 & 0.0464444 \\
\hline 0 & 0.1445338 & 0.1462684 & 0.3320093 \\
\hline 1 & 0.115851 & 0.114582 & 0.0443172 \\
\hline 2 & 0.0009083 & 0.0008311 & 0.0001173 \\
\hline
\end{tabular}

Table 4: The table is mean squared errors of three estimators of $I(\theta=0)$ when $m=3, r=1$ and $n=15$. The simulation is based on 3000 replicates.

\begin{tabular}{|c|c|c|c|}
\hline$\theta$ & MSE 1 & MSE 2 & MSE 3 \\
\hline-2 & $3.30 \times 10^{-10}$ & $3.29 \times 10^{-10}$ & $1.10 \times 10^{-12}$ \\
\hline-1 & 0.04351728 & 0.04351725 & 0.00096786 \\
\hline 0 & 0.01856288 & 0.01856291 & 0.329691 \\
\hline 1 & 0.03704426 & 0.03704423 & 0.001205216 \\
\hline 2 & $1.85 \times 10^{-9}$ & $1.85 \times 10^{-9}$ & $8.018 \times 10^{-12}$ \\
\hline
\end{tabular}

Table 5: The table is mean squared errors of three estimators of $I(\theta=0)$ when $m=0.01, r=1$ and $n=15$. The simulation is based on 3000 replicates.

\begin{tabular}{|c|c|c|c|}
\hline$\theta$ & MSE 1 & MSE 2 & MSE 3 \\
\hline-2 & $1.35 \times 10^{-15}$ & $1.34 \times 10^{-15}$ & $3.69 \times 10^{-13}$ \\
\hline-1 & 0.0008959 & 0.0008959 & 0.0010636 \\
\hline 0 & 0.346827 & 0.346827 & 0.3302176 \\
\hline 1 & 0.0007896 & 0.0007896 & 0.0010917 \\
\hline 2 & $1.108 \times 10^{-14}$ & $1.106 \times 10^{-14}$ & $3.91 \times 10^{-12}$ \\
\hline
\end{tabular}


From Table 2-5, we know that $m$ and $r$ can be selected to let $\eta_{1}(x)$ and $\eta_{2}(x)$ dominate p-value when $\theta=\theta_{0}$ or $\theta \neq \theta_{0}$. The selection of $m$ and $r$ can depend on the practical situations.

\section{References}

[1] Berger, J. O. and Wolpert, R. L. (1984). The Likelihood Principle. Institute of Mathematical Statistics, Hayward, CA.

[2] Casella, G. and Berger, R. L. (1990). Statistical Inference. Wadsworth \& Brooks/Cole Advanced Books \& Software, Pacific Grove, CA.

[3] Fraleigh, J. B. (1967). A First Course in Abstract Algebra,Addison-Wesley Publishing Co., Reading, Mass.-London-Don Mills, Ont.

[4] Herstein, I. N. (1975). Topics in Algebra, Second edition. Xerox College Publishing, Lexington, Mass.-Toronto, Ont.

[5] Hwang, J. T. and Casella, G. and Robert, C. and Wells, M. T. and Farrell, R. H. (1992). Estimation of accuracy in testing. Ann. Statist. 20, 490-509.

[6] Jacobson, N. (1985). Basic algebra. I. Second edition. W. H. Freeman and Company, New York.

[7] Lehmann, E. L. (1997). Testing statistical hypotheses. Reprint of the 1986 second edition. Springer-Verlag, New York.

[8] Schaafsma, W., Tolboom, J. and Van der Menlen, B. (1989). Discussing truth or falsity by computing a q-value. In Statistical Data Analysis and Inference (Y. Dodge, ed.) 85-100. North-Holland, Amsterdam.

[9] Wang, H. (2004). Improved estimation of accuracy in simple hypothesis versus simple alternative testing. Journal of Multivariate Analysis, 90, 269-281. 
[10] Woodroofe, M. and Wang, H. (2000). The problem of low counts in a signal plus noise model. Ann. Statist. 28, 1561-1569.

[11] Zwillinger, D., Krantz, S. G. and Rosen, K. H. (1996). CRC standard mathematical tables and formulae. 30th edition. CRC Press, Boca Raton, FL.

Received: March 15, 2006 